USO DEL FEEDBACK COMO ESTRATEGIA DE EVALUACION: APORTES DESDE UN ENFOQUE SOCIOCONSTRUCTIVISTA USING FEEDBACK AS EVALUATION STRATEGY: CONTRIBUTIONS FROM A SOCIOCONSTRUCTIVIST APPROACH

\author{
SECCIÓN ESPECIAL \\ Volumen 15, Número 1 \\ Enero - Abril \\ pp. 1-24
}

Este número se publicó el $1^{\circ}$ de enero de 2015

DOI: $\underline{\mathrm{dx} . \text { doi.org/10.15517/aie.v15i1.17633 }}$

Flor Jiménez Segura

Revista indizada en REDALYC, $\underline{\text { SCIELO }}$

Revista distribuida en las bases de datos:

CATÁLOGO DE LATINDEX, IRESIE, CLASE, DIALNET, DOAJ, E-REVIST@S, SHERPA/ROMEO, QUALIS, MIAR

Revista registrada en los directorios:

ULRICH'S, REDIE, RINACE, OEI, MAESTROTECA, PREAL, CLACSO 


\title{
USO DEL FEEDBACK COMO ESTRATEGIA DE EVALUACION: APORTES DESDE UN ENFOQUE SOCIOCONSTRUCTIVISTA USING FEEDBACK AS EVALUATION STRATEGY: CONTRIBUTIONS FROM A SOCIOCONSTRUCTIVIST APPROACH
}

\begin{abstract}
Resumen: El presente documento analiza el uso del "feedback" como una estrategia de evaluación desde una perspectiva constructivista sociocultural y situada, utilizado por un grupo de docentes de la educación superior en el Practicum. Se conceptualiza el feedback como una ayuda potencial del profesorado hacia estudiantado y que se distribuye a lo largo del proceso educativo en las situaciones de evaluación que se realizan; donde la persona docente puede ayudar en el ajuste y adaptación que experimenta cada una de las personas en dicho proceso. Esta investigación sirve como insumo para las personas profesionales en Orientación que se desempeñan como docentes y que pueden utilizar el feedback como una estrategia de evaluación. Algunos de los resultados de la investigación identifican su utilización en situaciones evaluativas por parte del personal docente del Practicum en el análisis institucional, la planificación, la programación, la intervención y su respectiva evaluación, la tutoría o seminario y la defensa ante un tribunal examinador de la memoria o carpeta de aprendizaje que realiza el estudiantado.
\end{abstract}

Palabras clave: FEEDBACK, PRÁCTICAS DE EVALUACIÓN, ENFOQUE SOCIOCONSTRUCTIVISTA.

\begin{abstract}
This document discusses the use of feedback, from the sociocultural constructivist and situated perspective, as used as an evaluation strategy by a group of Higher Education teachers in the Practicum. Feedback is conceptualized as student assessments made by faculty that are potential aids and are distributed throughout the whole teaching and learning process. The educator helps in the adjustment and adaptation experienced by students in their educational process. This research serves as an input for Counselor educators who can use feedback as an evaluation strategy. Findings identified the use of feedback in assessment situations of the Practicum, such as: institutional analysis, planning, programming, intervention and its evaluation, elaboration of a learning portfolio, and tutoring or seminar for the preparation of the defense of the Practicum report.
\end{abstract}

Keywords: FEEDBACK, FOCUS EVALUATION PRACTICES, SOCIOCONSTRUCTIVIST.

\footnotetext{
${ }^{1}$ Docente e Investigadora de la Escuela de Orientación y Educación Especial y del Instituto de Investigación en Educación. Universidad de Costa Rica. Doctora en Psicología Educativa, Maestría Gerontología, Licda. en Ciencias de la Educación con énfasis en Orientación.
}

Dirección electrónica: flor.jimenez@ucr.ac.cr

Documento recibido: 14 de julio, 2014

Enviado a corrección: 1 de agosto, 2014

Aprobado: 8 de diciembre, 2014 


\section{Introducción}

En la formación del estudiantado universitario un elemento clave del aprendizaje son las prácticas y, en este proceso, las prácticas de evaluación en que participa con otras personas con mayor experiencia profesional, como lo es la persona docente, de la que potencialmente recibe comentarios o feedback para aprender, dirigidos a señalarle la distancia entre lo que ya ha aprendido y aquello que le queda por aprender.

El feedback se conceptualiza como la información o comentarios facilitados por el profesorado al estudiantado, en relación con los aspectos de la interpretación, la comprensión o la ejecución de una tarea para lograr reducir las discrepancias entre los conocimientos que muestra actualmente y el logro de una meta de aprendizaje (Hattie y Timperley, 2007). El suministro de información explícita sobre las normas de evaluación y de feedback desde las "personas expertas" al alumnado, se lleva a cabo guiado por la expectativa de que estos últimos podrán utilizar la información para desarrollar su propia comprensión y reorientar la propia actividad al logro de los objetivos.

En consecuencia, para lograr que las alumnas y los alumnos se conviertan en personas activas del proceso de enseñanza y aprendizaje, se necesita algo más que conocimientos disciplinarios o incluso un acercamiento al alumnado. Los estudiantes y las estudiantes deben abandonar el papel pasivo de seguimiento de «las instrucciones» en procesos controlados por expertas y expertos académicos e implicarse activamente en la valoración, revisión y mejora del propio aprendizaje (Moreno y Pertuzé, 1998; Yee Fan y Wai Kwan, 2005).

Un mediador o mediadora clave en el proceso de enseñanza y aprendizaje y de evaluación del estudiantado es la persona docente, que utilizan dispositivos educativos diversos, concretados en formas de actuación específica, para definir las ayudas que van a permitirles la construcción del conocimiento o el aprendizaje. El estudiantado aprecia y demanda un buen feedback, pero, así como con la práctica de la evaluación en su conjunto, todavía hay muchos aspectos poco conocidos y problemas con la práctica del feedback. Un estudio (MacLellan,1996) desveló que 30\% de los estudiantes encuestados no había encontrado ayuda en el feedback y la mayoría aseguró haberlo utilizado sólo a veces. Estos resultados se suman a los de otra investigación, que encontró que el feedback a menudo no es comprendido por el alumnado (Lea y Street, 1998), y a un tercer sondeo que concluyó que el estudiantado no suele leer las notas que los docentes escriben en los trabajos evaluados (Bailey, 2009). Una razón que podría explicar también estos resultados es que el feedback se 
da demasiado tiempo después de que el trabajo ha sido realizado, cuando las personas ya no están interesadas y se encuentran centradas en otras tareas (Gibbs y Simpson, 2009; Hattie, 1987; Rust, O’Donovan y Price, 2005).

Partiendo de estos planteamientos se definió como objetivo del presente estudio describir y analizar la práctica del feedback que utiliza la persona docente en las prácticas de evaluación que realiza en el Practicum. Este estudio se llevó a cabo en la Universitat de Barcelona-España y el marco referencial fue la perspectiva constructivista sociocultural situada y auténtica.

La investigadora considera oportuno los resultados obtenidos en el presente estudio, para ser tomados en cuenta en el quehacer de las personas formadoras de orientadoras y orientadores. Asimismo, por los profesionales de Orientación que conforman los Comités de Evaluación en las instituciones del sistema educativo.

\section{Aproximación teórica}

\subsection{Perspectiva constructivista sociocultural situada y auténtica}

La perspectiva constructivista sociocultural de la enseñanza y el aprendizaje que guía el presente estudio, entiende la educación como una práctica social con fines de socialización e individualización, como un instrumento por el cual una sociedad intenta promover el desarrollo del estudiantado universitario por medio del aprendizaje de saberes culturales relevantes que respondan a sus intenciones educativas, las cuales se concretan en un currículo que incluye contenidos y saberes fundamentales para el desarrollo de las competencias necesarias para enfrentar diferentes situaciones en su quehacer profesional. Desde esta perspectiva, "las intenciones educativas se logran a partir de una ayuda sistemática, planificada y continuada por parte de los adultos o personas más preparadas" (Coll, 2001, p. 157).

Las finalidades generales de la educación son promover la construcción de la identidad personal, y socializar a las generaciones más jóvenes, que las sitúe de forma competente, participativa, activa y crítica en la sociedad.

La Educación Superior va más allá del logro de esas finalidades generales, extendiendo el logro de las mismas a campos de actividad y conocimiento específicos. En este caso, y tomando como referencia la perspectiva constructivista, el aprendizaje se da en interacción entre la persona docente, que guía, orienta o sostiene la actividad de aprendizaje del alumnado en la construcción personal de significados y la atribución de sentido al 
aprendizaje. La finalidad es que logre elaborar el conocimiento y las competencias propias de su campo y aprender a aprender.

Lo que los alumnos aportan al acto de aprender, su actividad mental constructiva, es un elemento mediador entre la enseñanza del profesor y los aprendizajes que llevan a cabo. Recíprocamente, la influencia educativa que ejerce el profesor a través de la enseñanza es un elemento mediador entre la actividad mental constructiva de los alumnos y los significados que vehiculan los contenidos escolares. (Coll, 2001 p. 179)

Se trata de una actividad cuyos componentes fundamentales deben contemplarse y explicarse gracias a las relaciones o influencias mutuas, que, en el nivel de la interacción entre la persona educadora y estudiantado, se explican en términos de construcción, mediación e internalización de los significados de los contenidos y de sentido del aprendizaje.

\subsection{El Programa de evaluación desde la perspectiva constructivista sociocultural}

Un primer análisis de las prácticas de evaluación está formado por un conjunto de situaciones o actividades de evaluación, integradas por una o varias tareas de evaluación, seleccionadas y diseñadas tomando en cuenta las características de las personas jóvenes estudiantes y de la propuesta educativa en que su actividad de aprendizaje se inserta. La persona docente planifica, organiza y diseña las diferentes situaciones de evaluación que se realizarán durante el proceso de aprendizaje y las plantea y distribuye a lo largo del tiempo de duración del curso. Las situaciones de evaluación que componen el programa tienen el propósito de identificar si el estudiantado ha adquirido las competencias y los conocimientos trabajados en el proceso de enseñanza y aprendizaje.

El Programa de Evaluación debe estar dirigido a todo el estudiantado y puede tener previsto realizar algunas variaciones en la secuencia para aquellas personas que lo necesiten, permitiéndoles no participar en algunas de las situaciones o actividades previstas o desarrollando otras nuevas y diferentes. El análisis del programa permitirá obtener una visión global de las diferentes situaciones de evaluación planificadas por la persona docente y las características y finalidades de cada una de las situaciones en el marco más amplio de los procesos de enseñanza y aprendizaje. 


\subsubsection{Las situaciones o actividades de evaluación}

Es el segundo de los niveles de análisis. Son episodios de la actividad conjunta de las secuencias didácticas en los que la actividad -planificada o efectivamente desarrollada- entre la persona que enseña y la que aprende, está presidido por el motivo común, y al menos parcialmente compartido, de mostrar o ayudar a mostrar los conocimientos que el alumnado ha adquirido sobre diferentes competencias y contenidos. La evaluación permitirá tener elementos para que la persona docente aplique el ajuste de la ayuda a lo largo del proceso en el que se presenta el cambio o innovación, en relación a la construcción del conocimiento del estudiantado (Coll, 2003; Mauri, Coll y Onrubia, 1999; Miras y Solé, 1990; Rochera y Naranjo, 2000).

En este segundo nivel las situaciones de evaluación según Coll, Mauri y Rochera, 2012; Mauri y Barberá, 2007; Mauri y Rochera, 2010; Rochera y Naranjo, 2007, pueden estar constituidas por actividades de diferentes tipos, como son:

- Las actividades de preparación de la evaluación (SAP): se refieren a aquellas actividades o tareas de evaluación que se llevan a cabo antes de la aplicación de las situaciones o instrumentos de evaluación mismos o propiamente dichos.

- Las actividades de evaluación propiamente dichas (SAE), entendidas como aquellas tareas de evaluación específicas que se deberán resolver en un tiempo, condiciones y situaciones determinadas.

- Las actividades de corrección / calificación (SAC), dirigidas a corregir y calificar las tareas que componen la actividad de evaluación propiamente dicha mediante la aplicación de los criterios de evaluación y de puntuación.

- Las actividades de comunicación (SAD), dirigidas a devolver y comunicar a los estudiantes los resultados de su aprendizaje, obtenidos en las tareas de evaluación.

- y por último las actividades de aprovechamiento (SAA), que se caracterizan por explotar didácticamente los resultados de la evaluación -errores y aciertos- en el marco de nuevas tareas.

\subsubsection{Tareas de evaluación}

Componen cada una de las actividades de evaluación y constituyen el tercer nivel de análisis de las prácticas de evaluación; es decir, el ámbito más micro de aproximación a las prácticas evaluativas. En este nivel se da el planteamiento de diferentes preguntas, ítems o 
problemas que responden, aborda o resuelve el estudiantado en el transcurso de una determinada situación de evaluación.

Es necesario que la persona docente informe al estudiantado sobre los nombres de las tareas de evaluación que deben realizar y los productos que se esperan de cada una de ellas, los contenidos conceptuales, procedimentales y actitudinales que se evaluarán, la exigencia cognitiva que debe aplicar cada estudiante según la situación o actividad de evaluación solicitada.

Para lograr una calidad de la educación es necesario querer innovar y transformar en los diferentes niveles de las prácticas educativas la evaluación, tener en cuenta las diferencias individuales, la coordinación entre el profesorado, contar con un enfoque en el que se tenga claridad de lo que se entiende por evaluación del aprendizaje del estudiantado, un programa que contenga el conjunto de situaciones o actividades de evaluación que despliegan la persona docente y el alumnado en un proceso de enseñanza y aprendizaje; situaciones o actividades de evaluación que permitan tanto la persona docente como a las personas jóvenes estudiantes desarrollar la actividad conjunta y que permita adquirir contenidos y competencias (Coll, Barberà y Onrubia, 2000).

\section{La ayuda al alumnado en las prácticas de evaluación. El feedback}

\subsection{El feedback desde la perspectiva constructivista sociocultural y situada}

El enfoque constructivista sociocultural y situado conceptualiza el feedback como una ayuda potencial del profesorado al estudiantado, que se distribuye a lo largo del proceso de enseñanza y aprendizaje en las situaciones de evaluación que realiza la persona aprendiz.

El elemento que queda por resolver es sin duda el de la contingencia, de la ayuda y la capacidad de uso de la misma por el estudiantado.

En donde la ayuda se ajusta y adapta al proceso de construcción que llevan a cabo los alumnos o aprendices; es decir, la que, teniendo en cuenta de manera dinámica lo que los alumnos son capaces de comprender y hacer en cada momento, cambia en tipo y grado en función de las contingencias, da apoyo en aquello que se necesita y cuando se necesita, y lo retira globalmente de manera progresiva para facilitar el uso autónomo y funcional de lo aprendido en situaciones cada vez más variadas y compleja (Coll, Onrubia y Mauri, 2008 p.36). 
Para utilizar el feedback como una estrategia de evaluación que permita a la persona docente universitaria realizar los ajustes necesarios a cada persona estudiante, es requisito esencial definir claramente los criterios de evaluación para cada tarea, donde cada uno tenga una comprensión significativa de los mismos y desarrolle un compromiso activo con el uso de la valoración en la mejora del aprendizaje. De hecho, se aboga por un proceso de evaluación en el que el alumnado participe en cada etapa, a fin de que entiendan realmente las necesidades complejas y contextuales de la praxis de evaluación, en particular los criterios y normas que se aplican, y cómo, gracias a ello, pueden mejorar su trabajo (Mauri, Coll y Onrubia, 1999; Rust, O’Donovan y Price, 2005).

Si el equipo docente y el estudiantado tienen claros los criterios de evaluación y el proceso de uso de los mismos para emitir un juicio de valor de los aprendizajes permitirá a los docentes y las docentes utilizar dispositivos y mecanismos que promuevan una influencia educativa eficaz, la que puede contribuir efectivamente a que el estudiantado y otros aprendices elaboren esquemas de conocimiento cada vez más ricos, complejos y válidos (Coll, Onrubia y Mauri, 2008) lográndose una construcción de significados por parte del alumnado de forma interna y externa, que le ayude a apropiarse de forma satisfactoria de los contenidos que las situaciones educativas tienen como objeto.

\subsection{Características del feedback}

Es conveniente que la profesora o el profesor comunique, desde sus primeros contactos con las personas que están en formación, la finalidad y utilidad del proceso y cuándo y cómo efectuará el feedback. El alumno y la alumna deben percibir que junto a la persona docente forman un equipo que tiene como objetivo su propio aprendizaje.

Al comienzo de una entrega de feedback, especialmente en evaluaciones globales del estudiante, es conveniente discutir y concordar con éste una apreciación del conjunto de su desempeño, comparándolo con objetivos bien definidos, que pueden o no estar escritos, pero que deben tener sentido para el docente y el alumno. Es recomendable solicitar al alumno una autoevaluación, efectuando preguntas abiertas, como ¿Qué crees que hiciste bien? ¿Qué crees que necesita mejoría? Las respuestas pueden generar una agenda para guiar la discusión entre ambos. Las recomendaciones del docente serán mejor recibidas si ambos comparten el diagnóstico de la situación (Moreno y Pertuzé, 1998, p. 58) 
Estos autores recomiendan que el feedback deber ser descriptivo, simple y objetivamente claro y centrado sobre la actividad o tarea concreta, en cuyo caso disminuye la carga emocional del estudiantado dado que se representa la situación como manejable. Asimismo, puede darse de forma escrita u oral. Por el contrario, si el problema es planteado como una apreciación del docente o la docente acerca de la persona de la alumna o del alumno es más probable que se dé una reacción negativa o defensiva del alumnado, lo que conduce a que se pierda el propósito educacional del feedback y se altere la relación docente y personas en proceso de formación.

\subsection{La reflexión sobre la tarea tomando en cuenta el feedback}

Para que el alumnado logre una práctica reflexiva sobre su aprendizaje en una tarea específica, el feedback debe responder a tres preguntas que deben plantearse tanto el docente y la docente de la universidad como el alumnado (Hattie y Timperley, 2007): ¿Dónde voy? (¿Cuáles son los objetivos? ¿Qué quiero lograr?); ¿Cómo voy? ¿Qué progresos se están haciendo hacia la meta?) ¿Y ahora? (¿Qué actividades debo llevar a cabo para progresar?). Los estudiantes y las estudiantes pueden aumentar su esfuerzo, especialmente cuando conduce a la realización de tareas más difíciles o apreciar las experiencias de mayor calidad en lugar de limitarse a hacer "más" ya que lo pide el profesor o la profesora. Es probable que se esfuercen cuando el objetivo perseguido es claro y el hecho de comprometerse con el cumplimiento de la tarea esté contribuyendo a garantizar el éxito.

Asimismo, es necesario que el estudiantado conozca las actividades que deben realizar y los criterios de evaluación que dirigirán las tareas vinculadas con dichos objetivos. Si el alumnado tiene conocimiento sobre lo que debe hacer, cómo y de qué forma serán evaluados, podrán orientar mejor su actividad para realizar acciones que les permitan cumplir con las metas. Los criterios de evaluación sirven para identificar si el alumno y la alumna ha sabido o no hacer su trabajo, si ha ejecutado la actividad con éxito, etc., en consecuencia es importante que los criterios sean explícitos y, para ello, cabe lograr que docente y personas jóvenes estudiantes operacionalicen, tomando todos estos aspectos en cuenta (Jorba y Casella, 1997, p. 26):

a) Nombrar las operaciones que caracterizan un trabajo dado y el grado de aceptabilidad de cada una de estas operaciones.

b) Formular esas operaciones de la manera más concreta posible para que los alumnos puedan hacerse una representación correcta de ellos. 
c) Explicitar su pertinencia con los objetivos más generales de la tarea, cualquiera que sea el objetivo puntual escogido.

Al compartir, estudiantado y la persona docente, los criterios de evaluación, el feedback permitirá que realice los ajustes necesarios para culminar la tarea de forma satisfactoria. Además, las metas de aprendizaje resultan más eficaces cuando los alumnos y las alumnas comparten con los docentes y las docentes el compromiso de lograrlas, debido a que se sienten propensos a solicitar y recibir dicho feedback. Por lo tanto, es necesario que el alumnado ocupe un papel central y activo durante todo el proceso, conozca lo que es un buen rendimiento y la meta a lograr. Además, es necesario que clarifique las diferencias entre su aprendizaje actual y el esperado. Para que el feedback sea satisfactorio, docente y alumnado deben sentirse parte de un equipo (Ende, 1983; Nicol y Mcfarlane, 2006; Stobart, 2010) de modo que el feedback resulte:
a) oportuno y esperado
b) basado en las observaciones efectuadas personalmente por la persona docente
c) referido a conductas susceptibles de cambio
d) descriptivo de la actividad y no valorativo de aspectos personales
e) referido a situaciones concretas y no a generalizaciones
f) referido a decisiones y actos y no a intenciones o interpretaciones
g) negociado con el alumnado

En suma, las condiciones de aprendizaje mejoran cuando el estudiantado ha recibido feedback en el tiempo adecuado, preferiblemente en cada una de las fases que el estudiante y la estudiante realizan para culminar la tarea y han gestionado los ajustes para hacer modificaciones que les permitan la autorregulación de su quehacer (Wiggins, 2010).

\subsection{Niveles en los que la persona docente puede ofrecer feedback al estudiantado}

El profesor y la profesora pueden llevar a cabo el feedback orientado a los cuatro niveles siguientes (Black y William, 1998; Gibbs y Simpsom, 2009; Hattie y Timperly, 2007):

3.4.1. Feedback sobre la tarea (FT). El feedback puede darse sobre una tarea o producto. A menudo se le conoce con el nombre de correctivo o de conocimiento de los 
resultados. Este feedback se centra en identificar para el estudiantado lo bien que se puede realizar o desempeñar una tarea, puede referir la exactitud, la pulcritud, el comportamiento o a algún otro criterio relacionado con el cumplimiento de lo demandado o producto. Puede solicitar al estudiantado que corrijan la información y/o incluyan más información (información diferente o información correcta).

El FT más utilizado por un $90 \%$ del profesorado se ofrece de forma verbal o escrita. Diferentes investigaciones han evidenciado que los comentarios por escrito son más eficaces que la nota numérica, debido a que favorecen el aprendizaje (Black y Wiliam, 1998; Butler, 1988; Crooks, 1988).

El FT es más significativo si es inmediato y específico, el rendimiento del alumnado mejora ya que el estudiantado todavía no ha iniciado una nueva tarea y puede resultarle relevante para avanzar en sus competencias.

Es oportuno que se promuevan estrategias que permitan a los estudiantes y las estudiantes recibir feedback sobre cada tarea, tales como, ofrecer feedback individual a un estudiante o a una estudiante particular en una tarea personal al finalizar la misma, o bien, ofrecer feedback grupal durante el proceso de ejecución de la tarea en pequeño grupo.

Los beneficios de la FT son que las personas jóvenes estudiante:

- Centraran su atención en las diferentes retroalimentaciones que reciban de los docentes y las docentes y valorarán el nivel de importancia sobre la tarea y la mejora de la misma.

- Recordarán elementos precisos de las diferentes características de la tarea durante el proceso de la elaboración de la misma.

- Desarrollarán habilidades genéricas al centrar su atención en el uso de esas habilidades y no únicamente en el contenido.

- De sentirán motivados a corregir errores de modo que puedan continuar su aprendizaje. 
- Se fijarán en elementos importantes del proceso de aprendizaje, al analizarlos con ayuda del feedback, y, por este medio, podrán desarrollar estrategias metacognitivas (Gibbs y Simpson, 2009).

3.4.2. Feedback sobre el proceso (FP). Este tipo de feedback es específico de los procesos subyacentes a la tarea. Desde un sentido superficial del aprendizaje, implica la adquisición, el almacenamiento, la reproducción y el uso del conocimiento y, por lo tanto, se refiere más que al feedback de la tarea en sí misma. Desde un entendimiento profundo del aprendizaje, la construcción del significado se refiere fundamentalmente a las relaciones, a los procesos cognitivos y a la transferencia a otras tareas más difíciles o novedosas (Zimmerman, 2000).

Una forma importante de FP se refiere a las estrategias de los estudiantes y las estudiantes para la detección de errores y, por lo tanto, a cómo se proporciona feedback a sí mismos. Estos errores pueden indicar la necesidad de buscar estrategias nuevas o elegir otras diferentes que les permitan ser más eficaces, así como determinar las necesidades de ayuda. Dependiendo de su motivación para perseguir el logro de los objetivos o disminuir la diferencia entre el conocimiento actual y el objetivo o meta deseados, se puede propiciar que el estudiantado participe en la identificación de los errores y en la elaboración o selección de estrategias de corrección adecuadas (Hattie, Biggs, y Purdie, 1996).

3.4.3. Feedback enfocado hacia la autorregulación (FR). El feedback del alumnado puede estar enfocado hacia el ámbito de la autorregulación, lo cual implica una interacción entre el compromiso con la tarea, el control del propio proceso y la confianza en la propia capacidad de aprender. Se centra en la forma en que la persona docente supervisa al alumnado, dirige y regula las acciones para que logren los objetivos de aprendizaje. También implica guiar la autonomía, el autocontrol, la autodirección y la autodisciplina por parte del estudiantado. El FR incluye también el pensamiento autogenerado, los sentimientos y las acciones que son planeado y adaptado cíclicamente para alcanzar los objetivos personales y que pueda guiar al alumno o alumna a la búsqueda, a la aceptación y a la incorporación de la información sobre el feedback recibido. Los siguientes aspectos del FR median la eficacia del feedback (Zimmerman, 2000): 
a) La perspectiva que potencia la capacidad de crear internamente feedback y autoevaluación.

b) La buena voluntad para invertir el esfuerzo en la búsqueda y en el trato de la información de feedback recibida.

c) El grado de confianza o de certeza en la exactitud de la respuesta del feedback.

d) La atribución acerca del éxito o fracaso, y

e) El nivel de competencia en la búsqueda de ayuda.

Lo más importante es que los estudiantes y las estudiantes conozcan cómo, cuándo y dónde pueden buscar el feedback de la persona docente que le facilite la autorregulación. También este tipo de feedback, si se lleva a cabo de una determinada manera, contribuye a crear un ambiente de aprendizaje en el que el estudiantado desarrolla la autorregulación (Hattie, Biggs y Purdie, 1996).

La autorregulación se aborda como un proceso inherente al proceso mismo de enseñanza y aprendizaje, que necesita de espacios de reflexión y de actuaciones o intervenciones educativas específicas del profesor para que el alumno progrese en el dominio de estas competencias. De acuerdo con este criterio, las actividades de enseñanza y aprendizaje se conciben, al mismo tiempo, como actividades de enseñanza y aprendizaje de la autorregulación. (Mauri, Colomina y de Gispert, 2008, p. 382)

El aprendizaje autorregulado no es una habilidad o una aptitud, es un proceso complejo en el que intervienen factores cognitivos, motivacionales, la conducta y el contexto, interrelacionados de forma flexible y dinámica para generar, mantener y modificar el comportamiento en correspondencia con los objetivos planteados (Pintrich, 2000). Este sistema de enseñanza y aprendizaje pretende que las personas aprendan por sí mismas, dirijan su propio aprendizaje, sean responsables, tengan una visión sistémica al analizar las interrelaciones del sistema, entiendan los problema de forma no lineal, tengan la capacidad para trabajar en equipo para reestructurar modelos mentales, para aprender de la experiencia y de los errores y para desarrollar la creatividad y la innovación.

En general, los estudios señalan las siguientes características para diferenciar al alumnado que autorregula su aprendizaje de los que no lo hacen (Corno, 1992 y Zimmerman, Kitsantas y Campillo, 2005): 
a) Conocen y emplean una serie de estrategias cognitivas que les ayudan a transformar, organizar, elaborar y recuperar la información.

b) Saben planificar, controlar y dirigir sus procesos mentales hacia el logro de sus metas personales (metacognición).

c) Presentan un conjunto de elementos motivacionales y emocionales de carácter adaptativo, tales como un alto sentido de la autoeficacia académica, la adopción de metas de aprendizaje y el desarrollo de emociones positivas.

d) Planifican y controlan el tiempo y el esfuerzo que van a emplear en las tareas y saben crear y estructurar ambientes favorables de aprendizaje, tales como buscar un lugar adecuado para estudiar y pedir ayuda académica a los profesores y las profesoras y a los compañeros y compañeras cuando tienen dificultades.

e) En la medida en la que el contexto lo permite, intentan participar en el control y la regulación de las tareas académicas.

En conjunto, son capaces de poner en marcha una serie de estrategias volitivas, orientadas a evitar distracciones externas e internas, que les ayudan a mantener su concentración, su esfuerzo y motivación durante la realización de las tareas.

Dado que la autorregulación se aprende, creando un sistema de enseñanza y aprendizaje adecuado se puede también enseñar a participar en el control y la regulación del proceso de enseñanza y aprendizaje. Por todo ello, no basta con estudiar el feedback per se, sino estudiarlo en el marco del conjunto del sistema de ayudas educativas que la evaluación canaliza. El objetivo es volver al estudiantado competente en el uso de las mismas y en su aprovechamiento para guiar su acción de mejora. En este caso, se comprende la práctica de la evaluación como un sistema, como un todo, en el que se integran ayudas diferentes, y que puede constituir en sí mismo, o no, el apoyo necesario para el aprendizaje de la autorregulación. Entendida ésta, en este caso, como el recurso que el estudiantado necesita para usar eficazmente el feedback para gestionar autónomamente la mejora de la propia práctica (Hattie y Timperley, 2007).

3.4.4. Feedback personal (FP). Es conocido como el gran esfuerzo que expresa positivamente y muy poco negativamente la evaluación y los efectos sobre el alumnado. Ofrece al estudiante y la estudiante información relacionada y convertida en esfuerzo, compromiso con los objetivos de aprendizaje, mejoramiento de la 
autoeficacia o entendimiento sobre la tarea. El feedback personal puede tener un impacto sobre el aprendizaje únicamente si conduce a los cambios del estudiantado en: el esfuerzo; el compromiso; los propios sentimientos de eficacia en relación al aprendizaje, o en las estrategias que usa para comprender las tareas. Si existe poca información de cómo realizar la tarea, el efecto se diluye y se ve influenciado el autoconcepto de la persona en formación (Carles, 2006; Mauri, Coll y Onrubia, 1999).

Los elogios dirigidos hacia los estudiantes y las estudiantes no favorecen su eficacia porque conllevan poca información en las respuestas que se les proveen y desvía su atención de la tarea, el logro y el aprendizaje. El elogio dirigido al esfuerzo, a la autorregulación, al compromiso o a los procesos relacionados con la tarea, al proceso y al rendimiento son más favorables porque le ayudan en su proceso de completar la tarea.

En síntesis, hay una distinción entre el feedback sobre la tarea (FT), sobre el proceso para lograr la tarea (FP), sobre la autorregulación (FR) y sobre el yo (FS). Diferentes autores señalan que (FS) es el menos eficaz, mientras que el (FR) y el (FP) son poderosos en términos de que profundizan en el procesamiento de la información, el control metacognitivo y el dominio de la gestión de las tarea y, por su parte, el (FT) es eficaz si la información de la tarea es útil para mejorar la estrategia de transformación o mejora de la autorregulación (Hattie y Timperley, 2007).

El profesor o la profesora, que utilizan el feedback como una estrategia de evaluación, ayudan al alumnado, no sólo a re-aprender (re-construir) determinados contenidos, sino también a lograr el control progresivo sobre el propio aprendizaje de las competencias, lo que es lo mismo, a incrementar su carácter intencional, propositivo, autorregulado y mediado culturalmente. La autorregulación y la autonomía del aprendizaje es una característica inherente a la actividad de aprendizaje en sí misma y, en consecuencia, debe ser objeto de enseñanza para incrementar el control consciente del proceso y contribuir a mejorar no sólo el resultado, sino el aprendizaje en sí mismo (Mauri, Coll y Onrubia, 1999; Mauri, Onrubia, Coll y Colomina, 2005).

\section{Metodología de investigación}

\subsection{Enfoque metodológico de la investigación}

La presente investigación de la cual se extraen los resultados que se presentan en este artículo se ubica en el paradigma cualitativo (Erickson, 1997; Flick, 2004). La adopción de 
este enfoque se caracteriza por la adecuación necesaria entre métodos y teorías; la toma en consideración de perspectivas diversas de los participantes; la capacidad de reflexión del investigador y la investigación misma; la variedad de enfoques y métodos; la comprensión de los fenómenos desde dentro como un principio epistemológico; la reconstrucción de situaciones como un punto de partida; y la construcción de la realidad como base.

La opción metodológica de estudio es la fenomenológica que tiene por finalidad última identificar, describir y comprender, mediante la triangulación de informaciones diversas y complementarias, el fenómeno objeto de estudio en toda su profundidad y en su contexto real sin alteraciones de los elementos a estudiar, como lo es, lo que declaran las personas docentes universitarias sobre el uso del feedback que realizan junto con el estudiantado en su quehacer; concretamente en su prácticas de evaluación del Practicum (Stake, 1995; Weinberg, 2002; Yin, 1984).

\subsection{Contexto y sujetos}

Los sujetos lo configuran 9 tutores del Practicum de formación del profesorado de la Universitat de Barcelona, que hubieran impartido la asignatura de Practicum II, y a quienes la investigadora consideró, de acuerdo con los criterios que se detallan más adelante, como informantes clave.

a) Tutores del Practicum de reconocido prestigio, que se hubieran involucrado en el desarrollo del mismo y a quienes la Facultad de Formación del Profesorado considera que son referentes indispensables para contribuir a la concreción definitiva de dicho Practicum.

b) Que fueran de diferentes áreas de formación y quisieran voluntariamente participar en el estudio.

\subsubsection{Procedimiento e instrumentos de recogida de los datos}

En una primera fase de la investigación: se procedió a: la revisión documental, curricular y bibliográfica; a la identificación de las personas docentes. Se elaboró una guía de preguntas para las entrevistas a las personas informantes del Practicum.

En la segunda etapa de la investigación: Las entrevistas realizadas a la personas informantes clave fueron registradas en audio, transcritas y analizadas por medio de la selección de pequeños fragmentos que permitieran dar respuesta a cada una de las preguntas de la entrevista, las cuales a su vez estaban relacionadas con el propósito del análisis, el cual era las categorías conceptuales de análisis de la evaluación y el feedback. 
En la tercera etapa de la investigación: Los datos recopilados fueron triangulados y analizados para dar respuesta a la fiabilidad y validez del presente estudio La teoría es otro elemento importante en ese proceso de triangulación, la cual se realizó al combinar diferentes métodos, en nuestro caso, la entrevista y el análisis de los documentos (Denzin, 1990; Taylor y Bogdan, 2010).

\subsection{Información recopilada y análisis}

\subsubsection{La práctica de evaluación en la situación de evaluación}

A continuación se presenta la información y el análisis de la misma de forma integrada, tomando como apoyo teórico la perspectiva constructivista sociocultural, auténtica y situada.

Las tareas de evaluación que realizan como práctica de evaluación las personas docentes del Practicum son: el análisis institucional, la planificación, de como máximo dos unidades de programación, la intervención y evaluación de la misma, la tutoría o seminario y la defensa ante un tribunal examinador de la memoria o carpeta de aprendizaje.

Después de identificar las tareas de evaluación, se presentaran los resultados encontrados en la presente investigación, a partir de los diferentes tipos de actividades que mencionan Coll, Barberà y Onrubia (2000), tales como, actividades de preparación (SAP), actividades de evaluación propiamente dicha (SAE), actividades de corrección (SAC), actividades de comunicación (SAD) y actividades de aprovechamiento (SAA), que fueron descritos en el marco teórico.

4.3.1.1. Actividades de preparación de la evaluación propiamente dicha al inicio del Practicum: todas las personas entrevistadas declaran que emplean momentos preparatorios de la actividad de evaluación propiamente dicha al inicio del Practicum, tales como la activación de conocimientos previos, ofrecer información sobre los apartados de la memoria o carpeta de aprendizaje y la observación de su contenido para que el estudiantado entienda el producto final del Practicum que deben entregar. Algunas ayudas que las tutoras y los tutores mencionan emplear en los momentos preparatorios al inicio del Practicum son que el estudiantado conozca el plan docente, tengan modelos de memorias efectuadas por otros educandos en otros cursos y pautas y guías del contenido de la memoria o carpeta de aprendizaje. La finalidad es ayudarles a representarse claramente el producto o resultado de aprendizaje y a conocer los criterios de evaluación del producto final del mismo. Se ofrecen menos guías del proceso a seguir en su realización y sobre el feedback que recibirán de la 
persona docente para ayudarles a mejorar, conocer lo que han alcanzado e identificar aquello que les queda por lograr.

4.3.1.2. Actividades de preparación de la actividad de evaluación propiamente dicha durante el Practicum: todas las personas participantes del estudio declaran que la evaluación es procesual, pero un aspecto trascendental es que el alumnado del Practicum pueda lograr establecer relaciones múltiples y complejas con la información recopilada. Las personas docentes desean que los estudiantes no se queden en una simple descripción de la información sobre la institución o el aula, las unidades de programación, la defensa de la memoria o la carpeta de aprendizaje, sino que les solicitan interpretarla y mostrar su reflexión sobre las mismas basada en criterios y planteamientos educativos bien fundamentados. Sin embargo, las tutoras y los tutores mencionan que a la mayoría del alumnado se les dificulta mucho seleccionar los datos relevantes e interpretar y analizar la realidad educativa siguiendo criterios teóricos y prácticos adecuados.

Por ello, la actividad de evaluación de la memoria o carpeta de aprendizaje se distribuye en tareas parciales, que tienen entidad en sí mismas y que se entregan como evidencias del progreso de aprendizaje del alumnado a la persona docente, para su valoración y recomendaciones de mejora. La distribución de la elaboración y entrega de esas evidencias las pacta cada tutora o tutor de acuerdo con un calendario que se establece con el estudiantado en la tutoría y respetando las indicaciones que plantea la persona docente del centro escolar. El feedback es continuo, según las personas docentes universitarias, proactivo - retroactivo y centrado en la tarea y, en particular, en los errores.

Habitualmente se da oralmente y por escrito y de forma individual o grupal, se promueven discusiones grupales que también facilitan la interpretación de la realidad que encuentra el alumnado durante el Practicum. Las tutoras y tutores señalan que el acompañamiento y seguimiento de todo el trabajo efectuado por las alumnas y los alumnos se realiza durante todo el tiempo que dura el Practicum. Las personas docentes o tutoras leen todos los documentos escritos por el alumnado, para la memoria final de prácticas les dan sugerencias sobre los cambios que deben llevar a cabo en el documento y les dan pautas de observación o análisis que les sirvan de apoyo para ejecutar la interpretación. Es importante que las personas jóvenes cuando realizan las modificaciones de los documentos solicitados por la persona docente, debe tener claro que el error se evalúa como elemento que facilita la mejora y la reflexión sobre el propio aprendizaje. Se aportan criterios y 
argumentos basados en las teorías y conceptos estudiados en la universidad que fundamentan la identificación de los errores realizada por la tutora y el tutor y permiten al alumnado comprender y compartir sus propuestas de mejora.

Asimismo, se promueve que la reflexión final y la corrección definitiva de esos errores o la elaboración de las mejoras las lleven a cabo los propios educandos, dando cuenta de que han comprendido e interiorizado esos criterios y que saben utilizarlos para mejorar sus actuaciones o intervenciones educativas, aprendiendo de y por sí mismos y gracias a la ayuda de las personas docentes. Por ese medio se espera que muestre su capacidad de gestión del propio proceso de aprendizaje y autorregulación. En cualquier caso, lo que se le pide que muestre de forma explícita es la reflexión realizada a lo largo de todo el proceso de aprendizaje. Algunos señalan lo referente a la ortografía, la redacción, entre otros, y por medio de símbolos que los estudiantes conocen, y con los que identifican la propuesta de cambios que deben tomar en consideración. Las devoluciones efectuadas por las tutoras y los tutores tienen una función formadora o autoreguladora.

4.3.1.3. Actividades de corrección de la actividad de evaluación propiamente dicha: La corrección no incluye la calificación y constituye en realizar propuestas de mejora que deben tenerse en cuenta en cursos posteriores del Practicum y su mejora. Las personas tutoras se muestran poco explícitas en la forma en que llevan a cabo la corrección y en los criterios de calificación.

El feedback se ofrece según las personas entrevistadas, centrado en cada una de estas tareas escritas - específicamente basado en los borradores, las elaboraciones iniciales y progresivas de dichas observaciones, análisis y programaciones - ofreciéndose de modo continuo, aunque no siempre de modo suficientemente inmediato, y centrado en errores o aspectos concretos de la tarea que deberían mejorarse, con un claro carácter retroactivo. El momento en que reconocen que ofrecen más feedback y de manera más personalizada ocurre durante la elaboración de la programación. El feedback de forma grupal es utilizado para comunicar al alumnado aspectos puntuales que todos deben saber.

Las docentes y los docentes afirman que de la programación valoran fundamentalmente la selección de los objetivos y contenidos de aprendizaje; el uso de metodologías que propicien la interacción entre iguales y con la persona docente y que impliquen a las alumnas y los alumnos en el aprendizaje. La reflexión sobre evaluación no se 
menciona o no en el mismo grado ni tampoco el papel de la misma en el desarrollo de competencias de regulación del aprendizaje.

Las personas docentes declaran que en la calificación final valoran positivamente tanto que el alumnado se implique de manera responsable en el centro, el aula y, en menor grado, en las tutorías de la universidad, como que demuestren capacidad de reflexión en la memoria sobre la práctica realizada. En ambos casos, el estudiantado debe mostrar que vinculan lo aprendido en la universidad con la práctica, proponen a las niñas y los niños actividades de calidad educativa y atienden a la diversidad.

4.3.1.4. Actividades de comunicación: según los datos identificados, las personas docentes afirman que comunican los resultados de la evaluación al estudiantado por escrito, haciendo uso de los documentos formales de comunicación del Practicum. Eventualmente dicen que hablan con el estudiantado sobre esos resultados y expresan que lo hacen siempre a petición de las y los estudiantes.

No obstante, es necesario aclarar que en el apartado de actividades de comunicación no está tan definida la información obtenida por el alumnado. Se considera que, si durante el Practicum las personas docentes tuvieran muy bien identificadas las actividades 0 situaciones de evaluación con sus respectivos procesos, al finalizar cada situación o actividad de evaluación podrían retomar el proceso seguido tanto por el alumnado como por la tutora o el tutor y, a partir de esto, dar una calificación sobre cada situación o actividad de evaluación, tratando de no dejar esta tarea hasta el final del Practicum.

Podemos señalar tres tipos de situaciones de comunicación de los resultados de aprendizaje al estudiantado: a) en la devolución que la tutora y el tutor realiza de cada documento que revisa del alumnado, b) en las tutorías, cuando las presenta públicamente y comparte las inquietudes que cada persona estudiante le plantea respecto de sus avances, o c) al finalizar el Practicum, ya sea en la defensa de la memoria o en una reunión posterior. Para que se dé uno u otro tipo de actuación es necesario que las personas tutoras brinden a las personas jóvenes estudiantes una evaluación sumativa con función pedagógica porque, se quiera o no, el Practicum debe ayudarles a conocer la acreditación obtenida y aquello que les queda por alcanzar. No obstante, según los datos muchas de las personas tutoras no tienen suficientemente sistematizados los criterios con los que se valoran diferentes aspectos que se evalúan en el Practicum y, en lo relativo a la evaluación sumativa final, tampoco 
declaran cómo pasan a elaborar el porcentaje que corresponde a la nota o calificación final, lo cual puede repercutir en que se presenten algunas incongruencias en la evaluación.

Las situaciones de evaluación relevantes del Practicum se ubican fundamentalmente en el durante, con el objetivo de identificar necesidades y ayudarles en sus dificultades, y al final del proceso.

La elaboración y defensa de la memoria es la única situación de evaluación del Practicum propiamente dicha y se ubica, como producto, fundamentalmente al final del proceso, cuando el alumnado entrega a la persona docente y la defiende en público. Sin embargo, la elaboración de la memoria contempla la realización paulatina de diferentes actividades o tareas de evaluación que el estudiantado desarrolla con apoyo de la persona tutora del Practicum y en el que debe dar muestra de lo aprendido.

Finalmente, respecto a la memoria, las personas tutoras no mencionan que incluyan en su práctica de evaluación actividades de aprovechamiento caracterizadas por explotar didácticamente los resultados de la evaluación -errores y aciertos- en el marco de nuevas tareas. Resultaría muy conveniente elaborar recomendaciones que repercutan en la mejora de actividades futuras y del escrito y recopilación de evidencias de reflexión e intervención práctica.

\section{Conclusiones}

$\checkmark$ Se analizó las prácticas evaluativas del Practicum a partir de la perspectiva constructivista sociocultural auténtica y situada. La cual señala la importancia de identificar las actividades de preparación de la evaluación, las actividades de evaluación propiamente dichas, las actividades de corrección y calificación, las actividades de comunicación y las actividades de aprovechamiento.

$\checkmark$ La práctica de evaluación de las personas docentes está presidida por un enfoque pedagógico, dirigido a promover una valoración continua y reguladora, formativa y formadora, situada y auténtica de la evaluación.

Las personas tutoras señalan como situaciones de evaluación diferentes actuaciones que el estudiantado lleva a cabo a lo largo del Practicum (por ejemplo: conocer y analizar el centro escolar y el grupo clase; elaborar e implementar intervenciones docentes, formar parte de otras tareas propias de los equipos docentes: reuniones de coordinación, claustro, entrevista con los padres) y de los documentos en que dan cuenta de dichas actuaciones y de la reflexión sobre las mismas. 
La utilización del feedback se centra en ayudar al estudiantado a identificar los errores que cometen en los documentos (de muy diferentes tipos) que entregan, para profundizar en la reflexión y lograr que mejoren. La referencia son los criterios proporcionados por las personas docentes para la elaboración de los mismos mediante explicaciones, pautas y documentos modelo que los estudiantes observan. Sin embargo, hay necesidad de revisar las características y el enfoque que las personas docentes dan al feedback de modo que el estudiantado pueda usarlo en la mejora de sus actuaciones en los centros y en el avance desde la descripción al análisis y a una mejor relación entre la teoría y la práctica.

$\checkmark$ Las personas docentes dan el feedback a los estudiantes centrado en la tarea, lo cual favorece que éste resulte más efectivo para el estudiantado y le ayuda a tomar decisiones concretas para ir mejorando y, a la vez, disminuir la carga emocional que representa la realización de dichas actividades.

$\checkmark$ Es oportuno que las tutoras y los tutores tengan claridad del tipo de feedback que van a dar en cada situación o tarea de evaluación, así como, los criterios de evaluación que dirigirán el feedback. Esto para que el alumnado conozca qué tipo de feedback se le dará a partir de cada tarea de evaluación.

$\checkmark$ La investigación realizada puede ser un insumo en la prácticas de evaluación que desarrollan las personas formadoras de profesionales de orientación, ya sea en la práctica supervisada, así como, en los diferentes cursos que se imparten. Donde el feedback que se brinde al alumnado sea identificado el tipo, los criterios de evaluación sobre los que se desarrollará y las formas de aplicarlo. Asimismo, tomar en cuenta la importancia de planificar las actividades de preparación de la evaluación propiamente dicha al inicio, actividades de corrección de la actividad propiamente dicha, actividades de comunicación y actividades de aprovechamiento.

\section{Referencias}

Bailey, Richard. (2009). Undergraduate students perceptions of the role and utility of written assessment feedback. Journal of Learning. Development in Higher Education, (1). Recuperado http://www.aldinhe.ac.uk/ojs/index.php?journal=jldheypage=articleyop=viewArticleypath $\% 5 \mathrm{~B} \% 5 \mathrm{D}=29 \mathrm{ypath} \% 5 \mathrm{~B} \% 5 \mathrm{D}=26$

Black, Paul, y Wiliam, Dylan. (1998). Assessment and Classroom learning. Assessment in Education, 5(1), 7-75. 
Butler, Ruth. (1988). Enhancing and undermining intrinsic motivation: the effects of taskinvolving and ego-involving evaluation on interest and involvement. British Journal of Educational Psychology, 58(1), 1-14.

Carles, David. (2006). Differing Perceptions in the Feedback Process. Studies in Higher Education, 31(2), 219-233. Recuperado de http://www.victoria.ac.nz/education/pdf/david-carless.pdf

Coll, César. (2001). Constructivismo y educación: la concepción constructivista de la enseñanza En C. Coll. J. Palacios y A, Marchesi (Comps.), Desarrollo psicológico y educación 2. Psicología de la educación escolar 2 (pp. 147-186). Madrid: Alianza Editorial.

Coll, César. (2003). Esfuerzo, ayuda y sentido en el aprendizaje escolar. Aula de Innovación Educativa, 120, 37-43.

Coll, César. (septiembre, 2009). Los enfoques curriculares basados en competencias y el sentido de aprendizaje escolar. En $X$ Congreso Nacional de Investigación Educativa (pp. 1-30). Conferencia magistral llevada a cabo en el Congreso Mexicano de Investigación Educativa - COMIE, Veracruz, México. Recuperado de http://www.psyed.edu.es/prodGrintie/conf/CC COMIE09.pdf

Coll, César, Barberà, Elena, y Onrubia Javier. (2000). La atención a la diversidad en las prácticas de evaluación. Fundación Infancia y Aprendizaje,(90), 111-132.

Coll, César, Onrubia, Javier y Mauri, Teresa. (2006). Tecnología y Prácticas Tecnológicas: Las TIC como instrumentos de mediación de la actividad conjunta de profesores y estudiantes. Anuario de Psicología, 38(3), 377-400.

Coll, César, Onrubia, Javier y Mauri, Teresa. (2008). Ayudar a aprender en contextos educativos: el ejercicio de la influencia educativa y el análisis de la enseñanza. Revista de Educación, 346, 33-70. Recuperado de http://www.revistaeducacion.mec.es/re346/re346 02.pdf

Crooks, Terence. (1988). The Impact of Classroom Evaluation on Students. Review of Educational Research, 58(4), 438-481.

Denzin, N. (1990). Interpretative biography. Qualitative Research methods (Vol. 1). London: Sage Publications.

Gibbs, Graham y Simpson, Claire. (2009). Condiciones para una evaluación continuada favorecedora del aprendizaje. Barcelona: ICE y Ediciones OCTAEDRO. Recuperado de http://www.ub.edu/ice/sites/default/files//docs/qdu/13cuaderno.pdf

Ende, Jack. (1983). Feedback in Clinical Medical Education. JAMA, 250(6), 777-781. Recuperado de http://medicine.emory.edu/education/academy-of-medicaleducators/documents/feedback 
Erickson, Frederich. (1997). Métodos cualitativos de investigación sobre la enseñanza. En Merlin C. Wittrock, La investigación de la enseñanza II: Métodos cualitativos y de observación (pp. 195-431). Barcelona: Paidós.

Flick, Uwe. (2004). Introducción a la investigación cualitativa. Madrid: Morata.

Hattie, John. (1987). Identifying the Salient facets of a Model of Student Learning: A Synthesis of Meta-Analyses. International Journal of Educational Research, 11(2), 187-212. Recuperado de http://eric.ed.gov/?id=EJ374471

Hattie, John, Biggs, John y Purdie, Nola. (1996). Effects of Learning Skills Intervention on Student Learning: A Meta-Analysis. Review of Educational Research, 66(2), 99-136.

Hattie, John y Timperley, Hellen. (2007). The Power of Feedback. Review of Educational Research, 77(1), 81-112.

Jorba, Jaume y Casella, Ester. (1997). La regulación y la autorregulación de los aprendizajes. España: Síntesis.

Lea, Mary y Street, Brian. (1998). Student Writing in Higher Education: and Academic Literacies Approach, Studies in Higher Education, 23(2), 157-172. http://www.informaworld.com/smpp/content $\mathrm{db}=\mathrm{all} \sim$ content $=\mathrm{a} 718864008$

Mauri, Teresa y Barberà, Elena. (2007). Regulación de la construcción del conocimiento en el aula mediante la comunicación de los resultados de aprendizaje a los alumnos. Revista Infancia y Aprendizaje, 30(4), pp. 483-497.

Mauri, Teresa, Colomina, Rosa y de Gispert, Inés. (2008). Diseño de propuestas docentes con TIC para la enseñanza de la autorregulación en la Educación Superior. Revista de Educación, 348, 377-399. Recuperado de http://www.revistaeducacion.mec.es/re348/re348 16.pdf

Mauri, Teresa, Coll, César y Onrubia, Javier. (1999). La evaluación de la calidad de los proceso de innovación docente universitaria. Una perspectiva constructivista. Revista de Docencia Universitaria, 1(1), 1-11. Recuperado de http://revistas.um.es/redu/article/view/3341/3241

Mauri, Teresa, Colomina, Rosa, Martínez, Catalina, y Rieradevall, María. (2009). La adquisición de las competencias de autorregulación. Análisis de su concepción y aprendizaje en diferentes estudios universitarios. Revista d'Innovació i Recerca en Educació, (2), 33-60. Recuperado de http://www.raco.cat/index.php/REIRE/article/viewFile/131528/181344

Mauri, Teresa, Onrubia, Javier, Coll, César y Colomina, Rosa. (2005) La calidad de los contenidos educativos reutilizables: diseño, usabilidad y prácticas de uso. RED. Revista de Educación a Distancia, (2). Recuperado de http://www.um.es/ead/red/M2/

Moreno, Rodrigo, y Pertuzé, Julio. (1998). Retroalimentación Técnica Fundamental en la Docencia Clínica. Boletín de la Escuela de Medicina, 27(1). Recuperado de http://escuela.med.puc.cl/publ/Boletin/Etica/Retroalimentacion.html 
Nicol, David y Macfarlane-Dick, Debra. (2006). Formative assessment and self-regulated learning: A model and seven principles of good feedback practice. Studies in Higher Education, 31(2), 199-218.

Pintrich, Paul. (2000). An Achievement Goal Theory Perspective on Issues in Motivation Terminology, Theory and Research. Contemporary Educational Psychology, 25, 92-104. Recuperado de http://www.unco.edu/cebs/psychology/kevinpugh/motivation project/resources/pintrich0 $\underline{0 . p d f}$

Rust, Chris, O'Donovan, Berny y Price, Margaret. (2005). A social constructivist assessment process model: how the research literature shows us that this should be best practice. Assessment $y$ Evaluation in Higher Education, 30(3), 231-240.

Stake, Robert. (1995). The art of case study research. California: Sage Publications.

Stobart, Gordon. (2010). Assessment fit-for-the-future. En IAEA Annual Conference 2010 (pp. 1-13). Tailandia: Institute of Education University of London.

Taylor, Steve J., \& Bogdan, Robert. (2010). Introducción a los métodos cualitativos de investigación. Barcelona, Paidós pp. 1-343.

Yee Fan, Silvia y Wai Kwan, Alice. (2005). Tang Communicating feedback in teaching practice supervision in a learning-oriented field experience assessment framework. Teaching and Teacher Education, 23(7), 1066-1085.

Yin, Robert. (1984). Case-study Research: Design and Methods. Beverly Hills, California: Sage Publishing.

Weinberg, Darin. (2002). Qualitative research methods. Oxford: Blackwell.

Wiggins, Grant. (2010). Feedback: How Learning Occurs [blog]. Recuperado de http://www.authenticeducation.org/ae bigideas/article.lasso?artld=61

Zimmerman, Barry. (2000). Attainment of self-regulation: A social cognitive perspective. En Monique Boekaerts, Paul R. Pintrich, y Moshe Zeidner (eds.) Handbook of selfregulation (pp.13-39). San Diego, California: Academic Press.

Zimmerman, Barry, Kitsantas, Anastasia y Campillo, Magda. (2005). Evaluación de la Autoeficacia Reguladora: Una Perspectiva Social Cognitiva. Revista Evaluar, (5), 1-21. Recuperado de http://revistas.unc.edu.ar/index.php/revaluar/article/view/537/477 